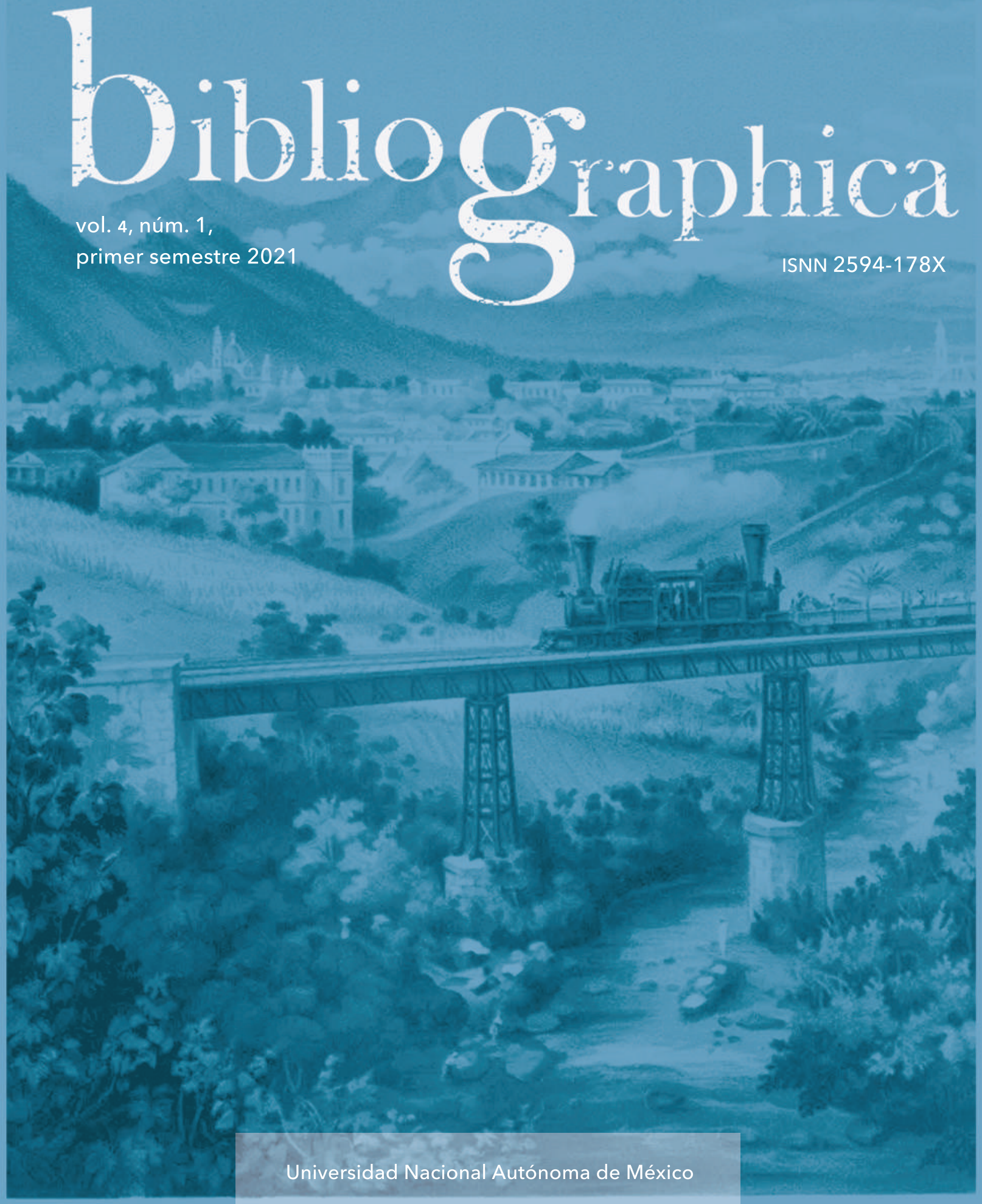




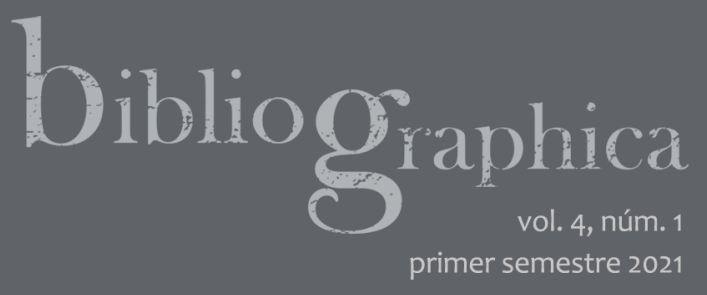

\title{
Manuales de investigación y referencias bibliográficas en México: el problema del sistema latino o hispánico tradicional
}

\author{
Research and Reference Handbooks \\ in Mexico: The Issue of the Latin \\ or Hispanic Traditional System
}

\section{Daniel Gutiérrez Trápaga}

Universidad Nacional Autónoma de México,

Facultad de Filosofía y Letras, Colegio de Letras Hispánicas,

Ciudad de México. México

dgtrapaga@hotmail.com

https://orcid.org/0000-0001-5203-6759

\section{Hugo Enrique del Castillo Reyes}

Universidad Nacional Autónoma de México,

Facultad de Filosofía y Letras, Colegio de Letras Hispánicas,

Ciudad de México. México

hugodelcastillo@filos.unam.mx

https://orcid.org/0000-0002-7345-1924

Recepción: 17.12.2020 / Aceptación: 12.02.2021

DOI: https://doi.org/10.22201/iib.2594178xe.2021.1.95 

tes en ámbitos académicos, para luego analizar los manuales bibliográficos y de investigación que han guiado y contribuido a la formación de investigadores en México desde la segunda mitad del siglo XX, y que siguen consultándose recurrentemente. Se trata de los textos de Bosch; De la Torre Villar y Ramiro Navarro; Galindo, Galindo y Torres-Michúa; López Valdés y el reciente manual de Esquivel Carreón, Rodas Rivera y Salazar Quintana, publicado por la Universidad Autónoma de Ciudad Juárez (UACJ). Los primeros cuatro describen, a veces sin nombrarlo, el sistema latino o hispánico tradicional; por ello examinamos el modelo de citas y bibliografía que presentan, a la luz de los criterios para evaluar normas bibliográficas propuestos por Lobato Domínguez. En su mayoría, estos manuales comparten dos rasgos que generan confusión sobre los sistemas para citar y elaborar el aparato crítico: no nombran el sistema que presentan ni refieren algún otro manual existente. Tampoco son críticos con el modelo que exponen, pues lo presentan como único. Lo anterior contribuye a crear la falsa idea de que existe un sistema latino o hispánico tradicional que se guía por los usos y costumbres, y no por un manual. Sin embargo, mostramos que el modelo base de dichos manuales y su supuesto sistema se asemejan a las normas ISO 690.

Palabras clave

Abstract

Keywords
Citas; bibliografía; sistema latino; sistema hispánico; ISO 690.

In order to underscore the relevance of the systematic referencing in academia, this work analyzes various bibliographic and research handbooks that have contributed to training researchers in Mexico since the second half of the $20^{\text {th }}$ century, and that remain in use. It focuses on those written by Bosch; De la Torre Villar and Ramiro Navarro; Galindo, Galindo and Torres-Michúa; López Valdés, and the recent handbook by Esquivel Carreón, Rodas Rivera and Salazar Quintana, published at UACJ. The first four describe, sometimes without naming it, the Latin or Hispanic reference system. We examine the reference guidelines they present in the light of Lobato Domínguez's criteria to evaluate systematization. Most of these handbooks share two confusing features: they do not name the reference system they present, nor they refer to any existing reference handbook or style. Furthermore, they are not critical of the guidelines they include, as they implicitly present theirs as the only existing reference system in academia. This creates the false idea that there is a traditional Latin or Hispanic system that is guided only by convention and not by precise guidelines. However, this work shows that the aforementioned handbooks guidelines resemble the ISO 690 reference standards.

Citations; bibliography; Latin system; Hispanic system; ISO 690. 


\section{Introducción}

Un aspecto fundamental de las técnicas y la metodología de investigación, particularmente en el caso de las humanidades, es el manejo sistemático de las fuentes de información, tanto primarias como secundarias. Con el enorme desarrollo tecnológico de las últimas décadas, este aspecto se ha vuelto cada vez más relevante ante la gran cantidad de datos disponibles acerca de casi cualquier tema. Las formas de organizar y consignar las fuentes de una investigación también son muy diversas, dependiendo de la disciplina y el tipo de publicación, así como de los múltiples sistemas que existen para este propósito.

Entre los sistemas de referencias bibliográficas, tres se han consolidado como dominantes en la investigación en humanidades: APA, a pesar de no tratarse de un sistema pensado para estas disciplinas; Chicago, tanto en su versión en notas a pie como en la de autor-fecha, y MLA. Estos sistemas se consignan en extensos manuales elaborados por las instituciones anglosajonas homónimas: la American Psychological Association, la University of Chicago y la Modern Language Association, que los actualizan periódicamente por medio de nuevas ediciones para adecuar dichos sistemas a los cambios de la investigación y sus fuentes, desde hace más de un siglo.

En consecuencia, diversos manuales de investigación, recursos didácticos e inclusive planes de estudio de las carreras y posgrados de humanidades exponen tales sistemas, o algún otro afín a las disciplinas del área. Además, no es extraño encontrar en esos recursos, al igual que en las facultades de humanidades del área de Letras en Colombia, España y, en particular, México, menciones o secciones dedicadas a un supuesto sistema adicional, que aparece con distintos nombres: "latino", "tradicional" o "hispánico". El objetivo de este trabajo es exponer y aclarar una serie de conflictos y confusiones respecto a ese supuesto sistema, su nombre y su manual de referencias, que ocasionan problemas a los investigadores y estudiantes de humanidades.

Uno de los manuales que expone y resume varios sistemas de referencias bibliográficas señala algunas de las características, retos y objeciones del sistema latino tradicional:

El sistema latino ha sido el modo de citar en la tradición editorial en español, y ha ido evolucionando a lo largo de los siglos conforme al desarrollo de los métodos, la tecnología editorial y las disciplinas académicas. En ese sentido, a diferencia de otros estilos de citación, el sistema latino se caracteriza por no 
tener reglas uniformes y normalizadas, pues sus modelos y formas han sido más resultado del uso que de la estipulación de reglas por parte de alguna entidad. Antes de que los sistemas de citación anglosajones empezaran a tener una marcada influencia en la edición de obras en español, hace algunas décadas, el sistema latino había sido el preferido y de mayor uso en lberoamérica. Al parecer, recibe el nombre de sistema latino por el uso frecuente de abreviaturas latinas (cf., ibid., idem, op. cit., entre otras) en la presentación de referencias dentro del texto. Sin embargo, este origen es incierto y en muchas ocasiones simplemente se denomina sistema tradicional de citación o por el nombre genérico de cita bibliográfica. ${ }^{1}$

Esta extensa cita causa una serie de preguntas que remiten a las definiciones más básicas de sistema y de investigación universitaria: ¿Cómo existe un sistema sin normas consignadas en un manual? ¿Cómo puede llamarse sistema, si carece de reglas uniformes y normalizadas? ¿Cómo ha evolucionado? ¿Quién o qué instituciones han estado detrás de dichos cambios? Incluso llama la atención que para los otros cinco estilos consignados en esta misma obra se remita a la fuente primaria, los manuales oficiales de cada sistema, así como a las instituciones académicas responsables de su elaboración, a diferencia de lo que ocurre en este caso.

Cabe destacar que los nombres que designan el sistema (latino o hispánico) manifiestan un elemento de nacionalismo académico, opuesto a los del mundo angloparlante que dominan la investigación, es decir, los referidos: APA, Chicago y MLA. Extraña, además, que no se tenga un nombre unívoco para referirlo, cosa que no sucede con ningún otro sistema de referencias y estilo, tanto en humanidades como en otras disciplinas. Lo anterior es un síntoma de los problemas estructurales que supone el uso de un sistema que aparentemente carece de manual, normas escritas y precisas.

La confusión en torno a este sistema lleva a suponer que pareciera aceptable y práctica común copiar la manera de citar de textos previos, en lugar de referirse al manual preciso que consigna el sistema. Luego, la descripción del sistema latino que ofrece el Manual de citas y referencias bibliográficas abre

\footnotetext{
1 Manuel Romero, "Sistema latino", en Manual de citas y referencias bibliográficas: Latino, APA, Chicago, IEEE, MLA, Vancouver, ed. de Manuel Romero et al., 2a. ed. (Bogotá: Vicerrectoría Académica, Universidad de Los Andes, 2015), 89. Este manual fue reeditado en México tres años después: Mercedes Perelló et al., Manual de citas y referencias bibliográficas, Biblioteca del Editor (México: UNAM / Universidad de Los Andes, 2018).
} 
otras preguntas que remiten a los principios básicos de investigación: ¿Cómo se puede sintetizar o exponer dicho sistema si no existe la fuente primaria? ¿Quién es el repositorio, almacén o soporte de la tradición a partir de la cual emana? ¿Al elaborar y revisar un texto, cómo puede saberse si se están respetando las normas, si se puso que no hay tales? ¿Qué sentido tiene utilizar un sistema con estos problemas para la investigación?

El presente trabajo examina a detalle la cuestión de este sistema de múltiples nombres, sin normas fijas o manual que lo consigne. Asimismo, buscamos aclarar el origen de esas confusiones y señalar la existencia precisa de una fuente clara, es decir, una serie de lineamientos propiamente consignados y detallados, en un texto elaborado por una institución internacional concreta. En particular, se revisarán diversos manuales de investigación y bibliografía que se publicaron y circularon ampliamente en México, los cuales han contribuido a crear -en mayor o menor medida- varias confusiones. Junto con la precisión en el manejo de fuentes, los siguientes criterios serán parte importante para evaluar las normas bibliográficas:

- Carácter unificador:

Es imperativo el consenso, su elaboración debe partir del debate entre un grupo amplio de profesionales y permitir de forma transparente -mediante los adecuados procedimientos de información pública- la participación de todos los colectivos implicados. Sin este acuerdo amplio se pueden dar recomendaciones, pero nunca normas [...].

- Seguridad o fiabilidad:

Debe partir de principios y métodos universales, de patrones o modelos probados. Para ello debe integrar diversas experiencias. Por esto, los organismos internacionales son los foros más adecuados para crear normas comunes y estables, al generar un clima de fiabilidad.

- Necesidad:

Debe servir para intereses colectivos generales y demandas de la sociedad. Es fundamental que esté orientada a los usuarios.

- Carácter finalista:

La norma debe resolver problemas, establecer metodologías y, en general, facilitar la vida (calidad) y economizar gastos.

- Accesibilidad:

La norma debe difundirse y conocerse, para poder ser aplicada. 
- Comprensibilidad (sencillez):

El aspecto lingüístico es un factor esencial en la normalización internacional. Términos precisos con relaciones unívocas entre los idiomas más extendidos.

- Tolerancia:

La norma no debe ser inmutable y fosilizar el ámbito de aplicación. Debe estar abierta a revisiones y cambios, pero también gozar de cierta estabilidad. ${ }^{2}$

\section{Ausencia de la fuente primaria y omisión de nombre del estilo o sistema bibliográfico}

Como se ha visto, el sistema que aquí revisamos carece de un nombre preciso, así como de un manual o normas que lo regulen, es decir, no tiene una fuente primaria para consultar sus reglas. Parece tratarse de una serie de prácticas que se heredan o copian de publicación en publicación. A diferencia de los otros estilos, no hay una organización o institución rectora que lo elabore y haga modificaciones con el tiempo. Luego, de inicio tenemos un problema de comprensibilidad, unificación y fiabilidad. En esta sección se revisarán algunos manuales sobre el manejo bibliográfico que, a partir de su configuración y presentación, han contribuido a la confusión en torno al sistema latino.

Dado que los sistemas para citar se cuentan en los miles, es frecuente que los manuales universitarios sólo expongan una acotada selección de ellos, o inclusive se limiten a uno solo. En el segundo caso, algunas publicaciones omiten señalar explícitamente cuál sistema presentan y que existen muchos más, aunque no estén dentro de los objetivos de determinados manuales. Así, encontramos cuatro textos de diversa índole, con múltiples ediciones, que generan una falsa idea sobre la existencia de un modo único de citar y presentar las fuentes empleadas en un trabajo: el libro La técnica de investigación documental, de Carlos Bosch García; La investigación bibliográfica, archivística y documental: su método, de Ernesto de la Torre Villar y Ramiro Navarro de Anda; el Manual de redacción e investigación, de Carmen Galindo, Magdalena Galindo y Armando Torres-Michúa, y la Guía de estilo editorial para obras académicas,

2 Javier Lobato Domínguez, "El nuevo marco normativo en Gestión de documentos de archivo, las normas ISO / UNE: Guía para profesionales", arch-e. Revista Andaluza de Archivos 1 (mayo de 2009): 4-5. 
de Mauricio López Valdés. ${ }^{3}$ Estas obras, todas con varias ediciones y utilizadas en el nivel universitario, servirán de calas en la forma en que se han expuesto y enseñado los principios de dicho sistema de citación.

Estos cuatro textos comparten otros rasgos importantes para la presente investigación: ninguno da nombre a las normas que consignan para citar, referir y elaborar la bibliografía. A pesar de que los cuatro presentan normas con notas a pie e incorporan los términos latinos como parte del aparato crítico, no las identifican de manera explícita con nombre alguno en el contenido presentado. Esa carencia lingüística complica la identificación del sistema e impide conocer el origen de estas normas, generando la idea -en un lector no especializado o en formación- de que sólo existe una manera de elaborar un aparato crítico. Es decir, si sólo se revisa un manual, se genera una falsa idea de unificación entre las maneras de citar y presentar la bibliografía. Si se consultan dos o más, el lector enfrenta variantes que producen problemas de unificación y fiabilidad, que también atentan contra el carácter finalista de las normas bibliográficas. En ese sentido, al carecer de una fuente primaria identificada de manera directa, pareciera que estos manuales exponen normas elaboradas por los autores a título individual y no tomadas de algún sistema, ya consignado debidamente en un manual, ya proveniente de normas copiadas o imitadas de publicaciones previas.

Por ejemplo, el manual de Bosch comienza con una advertencia en la que puede leerse lo siguiente:

Antes de comenzar creemos importante advertir a nuestros lectores que el presente trabajo se limita a una guía de tipo técnico que sirva para ayudar en la preparación de trabajos de clase, artículos científicos y tesis profesionales. Buscamos complementar las enseñanzas de los maestros con un instrumento que facilite la manifestación escrita de los conocimientos. [...] Si nuestro esfuerzo facilita un sistema para adquirir conocimientos concretos, para manejar y ordenar datos y para proceder a la meditación y al ejercicio de la razón, nos daremos por satisfechos. ${ }^{4}$

\footnotetext{
${ }^{3}$ Esta guía surgió originalmente para el área de publicaciones de la Facultad de Filosofía y Letras de la UNAM, donde se recogían sus fundamentos de estilo editorial. Mauricio López Valdés, Guía de estilo editorial para obras académicas (México: Ediciones del Ermitaño / UNAM, CRIM, 2009), 13-14; López Valdés, Guía básica de estilo editorial (México: UNAM, FFyL, 2003).

${ }^{4}$ Carlos Bosch García, La técnica de investigación documental, 3a. ed. (Caracas: Universidad Central de Venezuela, Instituto de Investigaciones Económicas y Sociales, 1972), 9. Se
} 
Aunque enfatiza que el manual tiene carácter de "guía técnica", no menciona los referentes consultados para elaborarlo. Además, al procurar "facilitar un sistema", no dice a cuál se refiere. De hecho, por como está redactada esa oración, parece que no existe sistema alguno y que el "esfuerzo" está en vías de dar sólo la posibilidad de elaborar uno.

Más adelante, dentro del apartado "Cómo lograr el material", Bosch explica cómo debe anotarse la ficha bibliográfica, mas no de dónde sale ese ordenamiento ni por qué; solamente se limita a poner ejemplos, alegando que:

La forma correcta de escribirlas es como sigue:

MacDonald, Austin F.

Latin American Politics and Government. New York, Thomas \& Crowell Co., 1949.

Esta sería una tarjeta bibliográfica típica para la bibliografía general, donde podría encontrarse relación con nuestro tema de historia internacional en caso de que este fuera el tema. ${ }^{5}$

Al referirse a la "tarjeta bibliográfica típica", queda claro que ese será el referente para toda la información que brinda, partiendo de una especie de usos y costumbres, idea que se fortalece al revisar que dicha "guía técnica" no consigna bibliografía alguna al finalizar su exposición. Así, Bosch expone la forma correcta y única para elaborar el aparato crítico: un sistema para referir la fuente en nota a pie, nuevamente sin mencionar el documento origen de lo expuesto (de haber consultado alguno), y tras ello explica el uso de los latinismos: opus citatus, ibidem, idem y locus citatus.

Poco más adelante, llama la atención la siguiente mención: "Algunos autores de trabajos norteamericanos citan al pie de página simplificando hasta el extremo. Ponen en su bibliografía la ficha completa como nosotros, pero en cambio al pie hacen mención solamente del apellido del autor y de la fecha de la edición seguida de la paginación de la cita: 10- $\underline{\text { Millares, }}$ 1958, p. 32". ${ }^{\prime 6}$ Bosch se refiere a "autores" que llevan a cabo esta práctica, pero no menciona que lo

consulta la tercera edición, pero se menciona que la primera es de 1968, además de que la primerísima estuvo a cargo de la UNAM en 1959, donde tuvo varias reediciones. Posteriormente se publicó en México decenas de ocasiones por la Editorial Trillas. La actual situación de pandemia de covid-19 impidió trabajar con alguna de las ediciones mexicanas. ${ }^{5}$ Ibid., 16.

${ }^{6}$ Ibid., 47. 
hagan por seguir otro sistema, lo cual parece lógico, pues hace énfasis en lo "norteamericano"; no queda claro si prefiere no decirlo o si quizá lo ignora.

Si bien el manual de Bosch tiene un apartado dedicado a "Bibliografía", ya no se habla más de normas para referir fuentes, pues sólo menciona la necesidad de consignar la misma información que ya se tiene en el fichero. Como dijimos, este manual no hace mención alguna de la existencia de varios sistemas para referir fuentes ni parece reconocer que se está describiendo uno, simplemente maneja la exposición como si sólo hubiera una manera correcta, lo cual es consistente con que no aparezca ni una sola fuente de consulta bibliográfica, a pesar de ser un manual que persigue el uso "correcto" de llevar a cabo investigaciones en humanidades.

El libro de De la Torre y Navarro muestra un problema similar. Al presentarlo, los autores hacen hincapié en su carácter de "obra metodológica", y apuntan: "La hemos elaborado guiados, lo reiteramos, por el pensamiento de notables educadores y por la propia experiencia nutrida en ellos y aplicada a la realidad educativa de nuestros países".7 Pero es de notar que no nombran a ningún educador en particular, ni de nombre ni de obra. ${ }^{8}$ Además, la idea final refuerza aquello de que la información proviene de la "experiencia propia", es decir, el recurso de la fijación de un conocimiento que bebe de los usos y costumbres. Esto lo justifica De la Torre Villar (si el discurso en primera persona se le adjudica) al decir que impartió la materia de "Métodos y técnicas de investigación" en diversas facultades de la Universidad Nacional Autónoma de México. Poco más adelante, vuelve a afirmar: "Nuestro texto, apoyado no sólo en la propia experiencia sino en muy respetados tratadistas, lo acompañamos de las notas de referencia correspondientes". ${ }^{9}$ Con lo cual afirma que el lector podrá encontrar las fuentes de donde se obtiene la información proporcionada, mas no es así en to dos los casos, como mostramos a continuación. Lo anterior refuerza la necesidad pedagógica de aclarar los puntos aquí tratados, para beneficio de la formación de los estudiantes de humanidades como investigadores.

En el apartado "El registro de las fuentes", De la Torre Villar y Navarro de Anda refieren el modo de consignar la fuente de un libro publicado: "Su for-

\footnotetext{
${ }^{7}$ Ernesto de la Torre Villar y Ramiro Navarro de Anda, La investigación bibliográfica, archivística y documental: su método (México: UNAM, 2003), xiv.

${ }^{8}$ Poco antes sólo refieren una brevísima lista de científicos al decir: "Paradigmas de hombres de ciencia fueron Pasteur, Claudio Bernard, Ramón y Cajal, Ignacio Chávez", pero ninguno es estrictamente un educador. Torre Villar y Navarro de Anda, ibid., xiii.

9 Ibid., xvi.
} 
ma de registro se ha ido precisando con el tiempo; hoy día, gracias al esfuerzo de numerosos y destacados bibliógrafos esa forma de registro se acepta internacionalmente en diversos congresos y reuniones especializadas, por lo que puede decirse que existe un consenso general respecto a ella". ${ }^{10}$ Pero no hay referencias a los bibliógrafos ni de los congresos y reuniones especializadas que mencionan, como si todos estuvieran ocupados en esa única manera de referir las fuentes.

Tras exponer las diferencias entre las fichas del catálogo de una biblioteca y las de uso común de cualquier investigador, los autores apuntan: "De todos modos, en el registro de los libros hay que seguir un orden, un método, que es el que señalamos a continuación y que procede de las reglas de catalogación adoptadas por la American Library Association, en su última edición".." Entonces aclaran que siguen el orden propuesto por una asociación estadounidense, pero no especifican hasta qué punto la información que exponen fue obtenida de allí. Ocurre algo similar cuando presentan la forma de consignar la fuente de una publicación periódica. Mencionan hasta cinco publicaciones especializadas en el manejo de este tipo de textos: $:^{12}$ usan la primera para definir qué son, y las demás sólo representan meros referentes en caso de haber mayor interés por parte del lector, pero sin especificar si la información expuesta proviene de dichas publicaciones.

Ante la búsqueda de una posible definición y las fuentes de donde proviene el sistema expuesto en este manual, es relevante abordar el apartado denominado "Aparato crítico", al cual se le asigna como función primordial servir "de mención a todas las fuentes utilizadas en la investigación que originaron la información que contiene el texto o discurso científico". Con ello es claro que sólo toman en cuenta el tipo de citación con nota, ya sea a pie o a final del documento, pues la otra función otorgada al aparato crítico tiene que ver con

\footnotetext{
$10 \mathrm{lbid} ., 36$.

11 lbid., 40.

12 Ibid., 65-66. David Grenfell, Publicaciones periódicas y seriadas. Su tratamiento en las bibliotecas especializadas (Washington, DC: Unión Panamericana, 1962); W. W. Varossieau, A Survey of Scientific Abstracting and Indexing Services (La Haya: FID, 1949); Theodore Besterman, A World Bibliography of Bibliographies, and a Bibliographical Catalogue, Calendars, Abstracts, Digest, Indexes, and the Like (Ginebra: Societas Bibliographica, 1955); Constance M. Winchell, Guide to Reference Books, 7a. ed. (Chicago: Chicago American Library Association, 1951); Winfred Gregory Gerould et al., Bibliography Union Lists of Serials in Libraries of the United States and Canada (Nueva York, 1943).
} 
consignar "todas aquellas ideas accesorias o secundarias que eliminamos del texto". ${ }^{13}$

Poco después, ligada directamente a la conformación del aparato crítico, aparece una sección titulada "Las locuciones y su empleo en el discurso científico", la cual inicia al informar que son de uso "tradicional" y se conservan en latín, independientemente del idioma principal del discurso que ayuden a elaborar:

Cabe advertir que estas locuciones latinas se emplean de ordinario en numerosas disciplinas científicas, tanto en las ciencias médicas como en las sociales, su uso es muy frecuente. En el campo del derecho su uso es continuo. El derecho público como el privado las emplea de ordinario. El derecho internacional abunda en expresiones latinas usadas tradicionalmente. Existen glosarios especiales de términos latinos que pueden consultarse con provecho. ${ }^{14}$

Llama la atención que los autores justifiquen el uso de locuciones latinas por lo aparentemente extendidas que están en varias disciplinas, sin ofrecer prueba de ello y que den preponderancia a su uso cotidiano en varias ramas del derecho, como si esto les otorgara mayor valía. También mencionan provechosos glosarios, pero no se refieren específicamente a ninguno. Tras lo cual explican el uso de los latinismos: et al., sic, circa, in margine, apud, conferre, ibidem, idem, loco citato, opus citatum, passim, infra, supra y vide.

Por último, es importante señalar que los autores presentan una sección final titulada "Bibliografía comentada de las obras referentes a métodos y técnicas de investigación bibliográfica, archivística y documental", donde consignan A Manual of Style de la Universidad de Chicago, novena edición, del que sólo mencionan: "Fue una de las obras más utilizadas en los medios universitarios de Estados Unidos". ${ }^{15}$ Y después aparece el MLA Handbook for Writers of Researchs Papers, Theses and Dissertations de 1977, del cual apuntan: "Sucinta guía con las indicaciones más someras en torno a la preparación de un trabajo escolar". ${ }^{16}$

Lo anterior indica que conocen la existencia, al menos, de estos dos sistemas y que no ignoran su relevancia, pues del manual de Chicago reconocen su uso extendido en Estados Unidos; sin embargo, está implícita una resistencia a considerarlos alternativas viables al sistema que exponen, lo cual queda en evi-

\footnotetext{
${ }_{13}^{13}$ Torre Villar y Navarro de Anda, La investigación bibliográfica, 152.

${ }_{14}$ Ibid., 156.

${ }^{15}$ Ibid., 274.

${ }^{16}$ Ibid., 278.
} 
dencia cuando, escuetamente, al manual de la MLA sólo le confieren valor para preparar trabajos escolares.

Por otro lado, el libro de Galindo, Galindo y Torres-Michúa presenta así lo concerniente a los sistemas de referencia y manejo bibliográfico:

La tercera y última parte se dedica a la investigación. Se incluyen modelos de fichas bibliográficas, de revista, de periódicos, de enciclopedias y antologías. Sin embargo, como la mayoría de las fuentes no se ajusta a los modelos, hemos desarrollado los principales problemas, acompañados de ejemplos, que presentan cada uno de los datos de las fichas, tales como la aparición de varios autores, títulos en otros idiomas, coediciones, etcétera. [...] En el capítulo 8 se resumen las diferencias entre fichas bibliográficas y notas a pie de página, así como una lista de fácil consulta de los términos usuales en investigación como op. cit., o ibídem, entre muchos otros. ${ }^{17}$

Este pasaje muestra cómo, al igual que en los anteriores manuales revisados, no identifican con un nombre o de manera clara qué sistema están siguiendo para la elaboración de las fichas bibliográficas o notas al pie. Si bien el pasaje habla de modelos, que inclusive son problemáticos, tampoco especifican a cuáles se refieren. Nuevamente, presentan una lista de términos latinos sin contextualizar su uso a partir de unas normas precisas. Como en los casos anteriores, esta situación resulta en problemas de comprensibilidad, unificación y fiabilidad, en caso de consultar un segundo manual.

Estos conflictos también pueden apreciarse en el interior del texto, al omitir información relevante sobres sus fuentes y sólo sugerirla. Al inicio de la referida tercera parte de este manual, aparece otro comentario sobre este tema: "Tanto las referencias bibliográficas en las notas a pie de página como la bibliografía obedecen a normas internacionales con la finalidad de identificar de manera precisa las obras utilizadas en la investigación y facilitar consultas subsecuentes a otros estudiosos". ${ }^{18}$ No aclaran a qué normas internacionales se refieren.

Si bien podemos suponer que hablan de las normas bibliográficas académicas ISO 690, este manual no lo aclara y tampoco cuenta con un apéndice bibliográfico que permita confirmar esta suposición. La ausencia de dicho

${ }_{17}$ Carmen Galindo, Magdalena Galindo y Armando Torres-Michúa, Manual de redacción e investigación (México: Grijalbo, 1997), 20.

18 Ibid., 203. 
apéndice termina por enfatizar algunos problemas ya señalados para los textos previos: generar la idea implícita de que sólo existe una manera de citar y de que se trata de una creación de los autores del manual en cuestión.

Por su parte, la Guía de estilo editorial para obras académicas, de Mauricio López Valdés, es aún más escueta en torno a las normas de referencia que incluye, reforzando la idea de que no existe una fuente previa y de que se trata de una guía con un sistema de citas de creación plenamente individual. El autor presenta así su manual:

Una primera versión de la obra la realicé en 2002 para la Facultad de Filosofía y Letras de la UNAM [...]. Me hice cargo del proyecto no sólo por ser quien estableció los fundamentos del estilo editorial de la Facultad hace casi veinte años, sino también con el afán de organizar y sistematizar diversos materiales que había yo elaborado [...], incorporando, asimismo, mis conclusiones emanadas de la indagación y análisis que de los diversos aspectos que conforman un estilo editorial he realizado en poco más de dos décadas. ${ }^{19}$

La propuesta de esta Guía en su totalidad es trabajo indudable del autor y contiene una extensa bibliografía, sin embargo, no menciona explícitamente un manual o textos de sistemas o normas bibliográficos. La Guía carece de un aparato crítico que vincule sus contenidos de manera clara con la bibliografía incluida. En el apartado titulado "Aparato crítico" se incluyen secciones dedicadas a la presentación de las notas al pie y la bibliografía, que detallan cómo organizarla, citar distintos tipos de fuentes, la información que debe consignarse y las abreviaturas que se emplean en el aparato crítico. ${ }^{20}$

Igual que los otros manuales, no nombra el sistema prescrito en el texto ni revela la fuente de las normas expuestas, presentándolo de manera implícita como creación del autor y como la única manera posible de citar. Si bien tras este manual hay un loable esfuerzo por unificar editorialmente las muy diversas publicaciones de la Facultad de Filosofía y Letras de la UNAM, este trabajo no está exento de los problemas señalados en los otros tres manuales: la omisión del nombre del sistema, la ilusión de que existe uno solo y que fue creado por el autor del texto.

\footnotetext{
${ }^{19}$ López Valdés, Guía de estilo editorial para obras académicas, 13.

${ }^{20}$ López Valdés, Guía básica de estilo editorial, 83-115.
} 
Los cuatro manuales analizados explican de manera directa la forma de citar, referir y hacer la bibliografía, pero sin dar más detalles sobre su fuente primaria o el estilo que proponen. No podemos dejar de señalar la paradoja de que estos manuales de investigación, que dedican importantes apartados al manejo de fuentes y bibliografía, tengan las deficiencias apuntadas. Sin duda este tipo de publicaciones ha contribuido significativamente a crear la idea sobre la existencia de un sistema de referencias en el mundo hispánico -por lo menos en el caso mexicano- que ha surgido de manera espontánea, sin ninguna institución o manual que lo norme. Es decir, quien utilice más de uno de estos manuales se enfrentará con problemas elementales de comprensibilidad, unificación y fiabilidad. Como veremos, efectivamente se trata de un sistema en común, pero proveniente de uno específico y consignado, aunque los manuales lo omitan, generando un falso problema de unificación.

\section{Las normas ISO como referentes centrales del estilo latino, hispánico o humanístico}

En la investigación llevada a cabo para encontrar manuales que normaran este sistema, encontramos uno muy reciente (2017, con una segunda edición de 2018) titulado Estilo Latino-humanístico. Redacción de textos académicos, editado por la Universidad Autónoma de Ciudad Juárez (UACJ) y elaborado por Luis Carlos Salazar Quintana, Guadalupe Esquivel Carreón y Beatriz Rodas Rivera. Para los propósitos de este análisis, vale la pena revisar algunas consideraciones hechas en este documento.

En su "Introducción" se afirma que es de herencia europea, lleva tres siglos funcionando y tiene tres nombres: sistema "tradicional", "latino-humanístico" o "cita-nota", ${ }^{21}$ y para estos datos remite a un par de artículos académicos de Gemma Muñoz-Alonso López, de los cuales se hablará más adelante. ${ }^{22}$ Es claro que los dos primeros apelativos pueden referirse a la práctica común que hemos mencionado, pero el tercero remite de inmediato a una cuestión

${ }^{21}$ Guadalupe Esquivel Carreón, Beatriz Rodas Rivera y Luis Carlos Salazar Quintana, Estilo Latino-humanístico. Redacción de textos académicos (Ciudad Juárez: UACJ, 2017), 9.

22 Gemma Muñoz-Alonso López, "Tendencias actuales de citación en los trabajos de investigación filosófica", Investigación Bibliotecológica 41, núm. 20 (diciembre de 2006): 91-106; "Citación y referenciación en el ámbito de la filosofía: personalización de estilos internacionales mediante gestores bibliográficos", ÉNDOXA: Series Filosóficas 31 (2013): 211-252. 
que está normada por otros sistemas (Harvard, Chicago, y en realidad cualquiera con notas a pie o finales), es decir, el tercer apelativo no es exclusivo de estas prácticas, por tanto, los autores se encargan de aclarar que el sistema adoptado usa latinismos de forma exhaustiva y, para ello, vuelven a citar a Muñoz-Alonso. ${ }^{23}$

Asimismo, los autores del manual de la UACJ afirman que "No hay manuales de referencias bibliográficas que desarrollen el sistema humanístico en español. Existen estilos anglosajones como Chicago y MHRA (Modern Humanities Research Association) que utilizan el sistema cita-nota con el uso de locuciones latinas, pero de forma limitada y no exhaustiva". ${ }^{24}$

Tan sólo en este estudio hemos expuesto cuatro manuales que presentan versiones de este modelo de referenciación en español, con el manejo exhaustivo de los latinismos. Como estos manuales no lo enuncian de manera explícita, no existe una sistematización a partir de fuentes en el libro Estilo Latino-humanístico, ya por ignorarlo, ya por creerlos sistemas distintos. Los autores de este libro piensan que están recogiendo las normas de uso sin conocerse o querer referirse entre sí, problema que ellos mismos advierten cuando aluden a "la imprecisión a la que se enfrentaban los usuarios de este estilo por no contar con un manual detallado". ${ }^{25}$

Dicho problema no se resuelve con este manual, pues los mismos autores se encargan de revestirlo con un uso local, al comentar que "la UACJ se ha inspirado en el estilo del Colmex y la UNAM, pero con sus propias consideraciones, por lo que el estilo de la UACJ es único y digno de ser documentado in extenso, en razón de las diversas publicaciones que genera la Universidad al amparo de esta forma de citación". ${ }^{26}$ En particular, se refieren a la Nueva Revista de Filología Hispánica y a la Revista de la Universidad de México. La primera tiene normas editoriales ("Lineamientos para publicar"), pero no un manual detallado que pueda constituir un sistema completo; ${ }^{27}$ la segunda, en cambio,

\footnotetext{
${ }^{23}$ Esquivel Carreón, Rodas Rivera y Salazar Quintana, Estilo Latino-humanístico, 9.

24 Ibid., 11.

${ }^{25} \mathrm{lbid}$.

${ }^{26} \mathrm{lbid}$.

27 Véase Colmex, "Lineamientos para publicar", Nueva Revista de Filología Hispánica, acceso el 13 de enero de 2021, https://nrfh.colmex.mx/index.php/nrfh/lineamientosparapublicar. En 1985 Luis Astey realizó otra breve propuesta para la Biblioteca Novohispana del Colegio de México; sin embargo, no se trataba de un manual para la formación universitaria y fue un texto que a la fecha sólo cuenta con una edición. Está dirigido a un ámbito muy especializado que es la ecdótica novohispana. De cualquier manera, el texto de Astey, si bien no lo enuncia de manera explícita, propone basarse en el sistema APA y no en un
} 
carece de lineamientos explícitos para sus colaboradores. ${ }^{28}$

Es relevante mencionar las referencias que el manual de la UACJ hace de Muñoz-Alonso, pues de allí obtienen los autores la información con la que presentan el "sistema" en su introducción. Muñoz-Alonso, en su artículo "Citación y referenciación en el ámbito de la filosofía: personalización de estilos internacionales mediante gestores bibliográficos" no explica el sistema, sólo menciona, al referir un ejemplo, que lo pondrá en "Sistema Tradicional de citación, denominado así porque hasta ahora ha sido el más utilizado en España; también se le conoce como estilo o sistema humanístico, europeo, francés, tradición hispánica o cita-nota". Y de inmediato refiere, en una nota a pie, que "El Sistema Tradicional se fundamenta en la Norma ISO 690 y 690-2". ${ }^{29}$ Esta misma información se lee en el manual de la UACJ (también en una nota a pie), pero allí se cita en el otro artículo de Muñoz-Alonso, en el cual sí se exponen las normas del "sistema tradicional", y para presentarlo se aclara lo siguiente:

Este sistema, también llamado cita-nota y a veces humanístico, se apoya en la norma internacional de descripción bibliográfica ISO, y son numerosos los países que aceptan y aplican la ISO 690-1987, International Standard Organization, en la norma Referencias bibliográficas: contenido, forma y estructura, y la ISO 690-2, parte $2^{a}$. En España asume ésta la Asociación Española de Normalización y Racionalización (AENOR), en la norma UNE 50-104-94; cuyo objetivo es indicar y organizar los elementos que han de colocarse en toda descripción bibliográfica, cualquiera que sea su soporte. ${ }^{30}$

modelo de nota a pie: "Tanto en los apéndices, cuando los haya, como en el texto y las notas de la o las introducciones y en la anotación general, las referencias a la bibliografía de apoyo: a) se indicarán cada una mediante una clave bibliográfica compuesta de 1) el o los apellidos del autor o los autores, 2) el año de publicación de la obra, y 3) una minúscula consecutiva, a partir de la 'a', si se usan dos o más obras que un mismo autor haya publicado dentro de un mismo año -clave seguida de 4) la numeración, con caracteres romanos en mayúsculas, del volumen correspondiente y/o, con cifras arábigas y, en su caso, con sólo un espacio como separación con respecto a la del volumen de la página o de las páginas inicial y terminal a que la referencia remita. [...] b) se vincularán a una bibliografía, en la que las claves queden ordenadas primero alfabéticamente por apellido de autor y luego cronológicamente -según año y letra minúscula, si este último es el caso- bajo cada apellido", Luis Astey, Procedimientos de edición para la Biblioteca Novohispana (México: Colmex, 1985), 27-28.

${ }^{28}$ Véase UNAM, "Colaborar en la revista", Revista de la Universidad de México, acceso el 13 de enero de 2021, https://www.revistadelauniversidad.mx/publish.

${ }^{29}$ Muñoz-Alonso López, "Citación y referenciación en el ámbito de la filosofía...", 215.

${ }^{30}$ Muñoz-Alonso López, "Tendencias actuales de citación...", 93. 
Así, tenemos declarado que el sistema "tradicional" sigue las normas ISO6901 y 2. Llama la atención que el manual de la UACJ no tenga estas normas citadas en su bibliografía ni las mencione en el cuerpo de su texto. ${ }^{31}$ Este texto tampoco apunta que las normas ISO-690 tuvieron una última actualización en 2010 y que la AENOR las publicó en español hacia 2013; sin embargo, los autores están de acuerdo en reconocer que su sistema se apoya en ellas, repitiendo lo dicho por Muñoz-Alonso.

Al revisar directamente las normas ISO citadas, aparece una afirmación relevante apenas inicia el documento, en la sección "Scope": "This International Standard does not prescribe a particular style of reference or citation. The examples used in this International Standard are not prescriptive as to style and punctuation". ${ }^{2}$ Se lee exactamente lo mismo en la norma española de la AENOR, documento que afirma, en su sección "1 Objeto y campo de estudio": "Esta norma internacional no prescribe un estilo concreto de referencia o de cita. El estilo y la puntuación de los ejemplos anotados en esta norma internacional no tienen carácter prescriptivo". ${ }^{33}$

Queda claro entonces que las normas ISO, a pesar de tener como objetivo estandarizar un procedimiento y una forma de referir las fuentes documentales, son flexibles, pues dejan un margen para el estilo, llámese uso de cursivas, versalitas, puntuación entre elementos, etcétera, de allí que hagan hincapié en que los ejemplos no son prescriptivos. Luego, la tolerancia dentro de la norma explica el gran número de variantes que hay entre los sistemas que siguen las normas ISO y, por ende, los diferentes estilos que existen o se identifican con el nombre de "sistema hispánico, latino y humanístico", como veremos más adelante. Inclusive, esto también permite que se mantenga el uso de los términos latinos en el ámbito de habla hispana, a pesar de haber sido descartados por otros sistemas, como Chicago, ${ }^{34}$ o instituciones como la Real Academia Española

${ }^{31}$ Esquivel Carreón, Rodas Rivera y Salazar Quintana, Estilo Latino-humanístico, 71.

32 International Standard ISO 690: Information and Documentation - Guidelines for Bibliographic References and Citations to Information Resources, 3a. ed. (Ginebra: ISO, 2010), 1. 33 Asociación Española de Normalización y Certificación, Información y documentación. Directrices para la redacción de referencias bibliográficas y de citas de recursos de información (Madrid: AENOR, 2013), 6.

${ }^{34}$ La decimoséptima edición de este sistema aún explica ciertos términos latinos, pero desaconseja su empleo: "Chicago discourages the use of ibid. in favor of shortened citations [...]. Chicago discourages the use of idem, recommending instead that the author's last name be repeated". The Chicago Manual of Style, 17a. ed. (Chicago: The University of Chicago Press, 2017), 759-760. 
(RAE), por su falta de claridad. ${ }^{35}$ Es decir, dificultan el uso, la comprensibilidad y el carácter finalista que deberían tener.

Antes de comenzar con la revisión específica de los elementos para referir fuentes entre los distintos manuales, es necesario revisar un par de juicios más de Muñoz-Alonso, que defienden la valía del "sistema tradicional". La autora señala que "tiene para muchas entidades y autores numerosas ventajas [...] con el sistema cita-nota o tradicional se pueden utilizar una gama muy amplia de locuciones latinas y de abreviaturas de gran tradición filosófica que enriquecen el escrito y le confieren un estilo elegante y a veces suntuoso". ${ }^{36}$ Este juicio, visto como ventaja, reporta un alto grado de subjetividad. Decir que los latinismos enriquecen y le dan elegancia o suntuosidad a un texto es una mera opinión, cuando el uso de estos términos sólo hace más complejo el manejo de las fuentes y dificulta la localización de la información para quienes desconocen el sistema. Además, un texto académico no necesita ser elegante y suntuoso, pues elementos de este tipo únicamente tienen cabida una vez que se ha privilegiado la claridad y calidad de la exposición y de la argumentación.

Muñoz-Alonso reporta otra ventaja de este sistema, pero comparándolo con Harvard, pues asegura que "presenta un nivel más alto de fidelidad de las fuentes", aspecto que le confiere al trabajo "rigor científico y utilidad para futuras investigaciones". ${ }^{37}$ Lo cual es válido, pero no es el único sistema que abona a ello, como es el caso de Chicago o MLA, sistemas pensados explícitamente para el trabajo científico de las humanidades y las lenguas. Finalmente, la autora expone que: "La desventaja del sistema humanístico deriva del abuso, a pie de página, de las locuciones latinas para indicar al lector los documentos y las páginas que apoyan el trabajo, produciéndose confusiones y paradas constantes en la lectura". ${ }^{38}$

En realidad, sólo la primera parte de esa "desventaja" es propia de este sistema. Salta a la vista que el mismo elemento señalado como ventaja es el que se vuelve una desventaja al hablar de su "abuso", cuando en realidad esto no

35 En el apartado "Sistema de notas", señala: "Dado que este sistema puede resultar incómodo y confuso para el lector, en especial si el trabajo es muy extenso o cuenta con muchas citas, es también posible emplear un método en el que la mención del autor y la obra se realiza de forma abreviada", RAE, Libro de estilo de la lengua española según la norma panhispánica (Barcelona: RAE / Espasa, 2018), párr. 92.

${ }^{36}$ Muñoz-Alonso López, "Tendencias actuales de citación...", 96-97.

${ }^{37} \mathrm{lbid} ., 97$.

${ }^{38} \mathrm{lbid}$. 
es una decisión de quien adopta el sistema, sino una regla de su uso que tanto Chicago como la RAE desaconsejan explícitamente. La segunda parte, en la que refiere la interrupción de la lectura, ocurre en realidad con cualquier sistema con notas a pie o finales, y lo mismo sucede con la última desventaja que reporta Muñoz-Alonso: "la unión de citas y de notas, [...] recarga innecesariamente algunas investigaciones, y les da una falsa apariencia de erudición". ${ }^{39}$ Ninguno de estos juicios se reportan en los manuales que hemos referido. Ahora es prudente pasar a la comparación de los elementos necesarios para referir una fuente, según los diversos documentos abordados.

\section{Comparación de la información y presentación de la bibliografía en los manuales consultados}

Dos aspectos centrales para el manejo bibliográfico y sus diversos sistemas son la información de las fuentes que debe consignarse en los aparatos críticos y el orden en que dicha información debe ser presentada. En términos generales, estos aspectos son muy similares en los diversos sistemas que existen para el trabajo en humanidades y otras disciplinas, pero las variaciones de tales aspectos son las que permiten distinguir los sistemas y elegir uno sobre otro.

Basta, por ejemplo, pensar en las diferencias que hay entre dos sistemas parentéticos, como APA y MLA, en la medida en que el primero incluye la fecha de publicación en los paréntesis de referencia. Esto resulta en cambios en la manera de presentar la información en la bibliografía, pues en APA la fecha aparece segunda tras el nombre del autor y antes del título, convirtiéndose así en el segundo criterio de alfabetización, en lugar del título de la publicación. En cambio MLA, como muchos otros sistemas, consigna la fecha de publicación, pero al final de la ficha bibliográfica, no en los paréntesis. Más allá de este ejemplo, a continuación revisamos la manera en que los sistemas de los manuales ordenan la información de sus fuentes, para mostrar que tienen como base las normas flexibles ISO.

En primer lugar, presentamos los elementos y el orden que marca la norma ISO 960-2010:

a) name(s) of creator(s), if available;

b) title;

\footnotetext{
39 Ibid.
} 
c) medium designation, if necessary;

d) edition;

e) production information (place and publisher);

f) date [in the name and date system (see Annex A), the year should not normally be repeated in this location unless a fuller date is necessary (e. $g$. for a serial)];

g) series title, if applicable;

h) numeration within the item;

i) standard identifier(s), if applicable;

j) availability, access or location information;

k) additional general information. ${ }^{40}$

Por su parte, el manual de la UACJ es más específico con la puntuación, pues está buscando declarar un estilo:

1. Se indica el nombre del autor y sus apellidos, seguidos de coma.

2. Título de la obra en letras cursivas, seguido de punto. Tomar en cuenta que cuando dentro del título se alude a una obra en particular, ésta debe ir en redondas y el resto del título en cursivas (vid. sección Cursivas).

3. Si hay colaboraciones, además del autor, éstas se tienen que señalar entre paréntesis, separando cada una con punto y coma. Asimismo, después del paréntesis se escribirá un punto: (ed.; trad.; pról.; intr.; etc.).

4. Nombre de la casa editora, seguido de coma.

5. Ciudad en que se edita la obra, seguida de coma.

6. El número de la edición ( $v$. g. $3^{a}$ ed.) seguido de coma; si tiene reimpresión esta deberá agregarse (v. g. $3^{a}$ ri.), seguida de coma. Es importante considerar que las primeras ediciones no se señalan, toda vez que se sobrentienden.

7. Año de edición, seguido de coma.

8. Para los volúmenes se usa la abreviación "vol." (v. g. 3 vols. para indicar una obra compuesta por 3 volúmenes; vol. 3 para indicar sólo el volumen 3), seguida de coma.

\footnotetext{
${ }^{40}$ International Standard ISO 690, 4. Cabe reiterar que esta información corresponde a la tercera edición de la guía ISO, ya adaptada a los formatos digitales. De cualquier manera, la información no es nueva, pues este organismo internacional data de 1946 y tiene antecedentes importantes que se remontan a inicios del siglo XX, Javier Lobato Domínguez, "El nuevo marco normativo en Gestión de documentos de archivo...., 6.
} 
9. Se señala el número total de páginas o, en su caso, las páginas donde se encuentra el texto citado.

10. Si el libro es parte de una colección o serie, se indica el nombre de estas al final de la referencia enmarcada en corchetes, sin agregar las palabras "serie" ni "colección", sino directamente la nomenclatura correspondiente; después de este registro debe señalarse una coma y el número que corresponda a la serie o colección. ${ }^{41}$

Varias páginas más adelante, se menciona que: "La estructura para presentar la bibliografía es la misma que se utiliza en las referencias a pie de página. La diferencia consiste solo en lo que atañe a la consignación del autor o autores, en virtud de que se invierte el orden del registro, esto es, se escriben primero los apellidos y después los nombres, separándolos entre sí por medio de una coma". ${ }^{42}$ Como puede observarse, se cumple con los elementos dispuestos por la norma ISO y siguiendo casi el mismo orden. A continuación, exponemos cómo los manuales mexicanos, referidos en la primera sección, se guían por las mismas categorías y orden de exposición.

En el caso del manual de Bosch, habla del sistema de referencias, sin decirlo explícitamente, en el apartado "Notas a pie de página", donde detalla la información que debe registrarse y el orden para hacerlo en la nota a pie, que sigue un formato corto de Autor, Título, página:

Al final del libro pondremos una bibliografía completa de todo lo utilizado. Cada palabra y cada renglón de impreso suma y aumenta el costo del libro. Por lo tanto debemos usar el menor espacio posible en el aparato accesorio de las notas. Pero no debemos prescindir de la información siguiente: 1) nombre o inicial del autor, 2) apellido paterno sin abreviaturas, 3) título del libro, completo si es corto o abreviado si es largo y siempre subrayado para que la imprenta lo forme en el tipo conveniente, 4) el tomo o volumen, siempre abreviado con el punto que indica la abreviatura y el número del mismo, 5) la palabra "página" en singular o en plural, pero siempre abreviada, 6) el número de la página a la que se refiere y 7) el punto que cierra la nota. Queda entonces la nota como sigue: 1. Rómulo Carbia, Crónica oficial de las Indias, p. $152 .{ }^{43}$

\footnotetext{
${ }^{41}$ Esquivel Carreón, Rodas Rivera y Salazar Quintana, Estilo Latino-humanístico, 36-37.

42 Ibid., 81.

${ }^{43}$ Bosch García, La técnica de investigación documental, 44.
} 
Para la bibliografía no señala de manera explícita las categorías a presentar, si bien pueden deducirse de manera sencilla por medio de sus ejemplos, como el citado previamente de la sección "Cómo lograr el material":

MacDonald, Austin F.

Latin American Politics and Government. New York, Thomas \& Crowell Co., $1949 .{ }^{44}$

En el ejemplo de lo que llama "tarjeta bibliográfica típica", puede verse que sigue el orden: Apellido, nombre. Título. Lugar de publicación, Editorial, año. Poco después, en el caso de la revista sólo explica que el título va entre comillas, para diferenciarla de la entrada de libro, y pone el siguiente ejemplo:

Verlinden, Charles.

"Santa María la Antigua del Darien, première 'Ville' coloniale de la Terre Ferme Americaine". Revista de Historia de América. México, Instituto Panamericano de Geografía e Historia, núm. 45, junio 1958, pp. 1-48. ${ }^{45}$

El ejemplo sigue el esquema: Apellido, Nombre. "Título del artículo". Nombre de la revista. Lugar de publicación, Institución a la que pertenece la publicación, número (núm. x), Mes año, número de páginas que abarca el artículo (pp. $x$-y). Es decir, Bosch sigue las categorías y el orden propuesto por las normas ISO.

Por su parte, el señalamiento de De la Torre y Navarro se hace de manera más directa, sin tener que inferir de los ejemplos la presentación de la información que debe incluirse para el aparato crítico:

Elementos indispensables para distinguir con rigor una obra de otra:

1. Nombre de autor.

2. Título y subtítulos completos.

3. Nombre del prologuista.

4. Número de volúmenes y edición.

5. Pie de imprenta constituido por el lugar de impresión, imprenta o casa editorial y fecha.

\footnotetext{
${ }^{44}$ Ibid., 16.

${ }^{45}$ Ibid., 18.
} 
6. Número de páginas e ilustraciones, e indicaciones de si contiene ilustraciones, mapas, gráfica, etc.

7. Colección o serie a que pertenece. ${ }^{46}$

A su vez, el Manual de Galindo, Galindo y Torres-Michúa sigue exactamente el orden y las categorías planteadas por De la Torre y Navarro, aunque la manera de agrupar los datos en incisos es distinta:

Los datos que se incluyen en la ficha bibliográfica son:

a. Nombre completo del autor.

b. Título de la obra, subrayado.

c. Editor, prologuista y/o traductor de la obra, si es necesario.

d. Número total de volúmenes si son más de uno.

e. Número de edición.

f. Pie de imprenta o datos de publicación que son tres: lugar de publicación, casa editora, año de publicación.

g. Número del volumen consultado, si no se leyeron todos.

h. Número total de páginas.

i. Nombre y número de la colección si la hay. ${ }^{47}$

El único cambio que destaca es la aclaración del inciso " $\mathrm{g}$ ", en caso de sólo haber revisado un volumen. Esta variación es menor y se trata de una precisión que no basta para considerarla un sistema distinto, por lo menos en lo concer-

${ }^{46}$ Torre Villar y Navarro de Anda, La investigación bibliográfica, 36. Más adelante especifican, con base en el mismo modelo, las variaciones para publicaciones de información general, "1. Nombre del autor. 2. Título del artículo o de la información (debe entrecomillarse). 3. Publicación en la que aparece (subrayado el título). 4. Fecha o fechas de aparición del artículo o información. Estos elementos bastan para el registro de artículos o de información procedente de periódicos comerciales, informativos, no científicos", y las variaciones para periódicos científicos: "1. Autor: síganse las normas ya señaladas. 2. Título: entrecomillado para diferenciarlo del título de la publicación. 3. Título de la publicación periódica en que aparece: subrayado o con tipo diferente. 4. Época, tomo, volumen o año, número, fecha de publicación y páginas en donde se localiza", 72-74.

${ }^{47}$ Galindo, Galindo y Torres-Michúa, Manual de redacción e investigación, 203-204. Igual que el manual de De la Torre y Navarro, este texto incluye la presentación de la información para otras fuentes, por ejemplo: "Modelo de ficha de revistas: a) Nombre completo del autor, si lo hay. b) Título del artículo, entrecomillado. c) Nombre de la revista, subrayado. d) Volumen y número si los hay. e) Fecha o entrega. f) Números de páginas en que se encuentra el artículo". 
niente a la información bibliográfica a registrar, su orden y la manera de presentarla. De hecho, la obra escrita por De la Torre y Navarro detalla este asunto en una breve sección titulada: "Número de volúmenes y de edición". ${ }^{48}$ De lo anterior se concluye que la aparente variación entre estos manuales se reduce a la forma en que exponen la información y no a una diferencia real en el contenido. Únicamente los manuales de Galindo et al. y de la UACJ agregan el número total de páginas de una publicación en cada entrada bibliográfica.

Con estos precedentes, no extraña que la Guía de estilo editorial de López Valdés proponga las mismas categorías y el mismo orden que los manuales expuestos:

1. Autor $[\ldots]$.

2. Título $[\ldots]$.

3. Subtítulo [...].

4. Aclaración al volumen [...].

5. Número de edición [...].

6. Tipo de edición [...].

7. Traducción.

8. Selección, prólogo y notas [...].

9. Introducción, estudio preliminar.

10. Pie de imprenta (ciudad, editorial, año) [...].

11. Páginas totales (sólo cuando se trata de un volumen) [...].

12. llustraciones, fotografías.

13. Colección o serie, entre paréntesis [.... ${ }^{49}$

Esta ficha es más extensa, pues contiene posibilidades no consideradas por los autores previos (consignar ilustraciones y fotografías) y es más explícita al desarrollar las posibles categorías a consignar entre el título y el pie de imprenta. Nuevamente, se trata de variaciones mínimas, principalmente en la manera de organizar los incisos en la Guía, que producen cambios menores en el trabajo con la información de las fuentes.

El alto grado de similitud de las normas presentadas directamente en los cuatro manuales, así como implícitamente en el de Bosch, se aprecia en las fichas

\footnotetext{
${ }^{48}$ Torre Villar y Navarro de Anda, La investigación bibliográfica, 59.

${ }^{49}$ López Valdés, Guía de estilo editorial para obras académicas, 88-89.
} 
con las cuales ejemplifican. Los tres primeros manuales siguen un mismo modelo que coincide no sólo en el orden y la información, sino hasta en la puntuación.

En el caso de Bosch, aparece la siguiente entrada bibliográfica de un libro:

Creel, George. The people next door. New York, The John Day Co., 1926. ${ }^{50}$

Por su parte, De la Torre y Navarro consignan el siguiente ejemplo:

Garcés, Jorge.

Paleografía, diplomática española y sus peculiaridades en América.

Quito, Publicaciones del Archivo de la Ciudad, 1949. ${ }^{51}$

En cuanto al Manual de Galindo, Galindo y Torres-Michúa:

Marx, Carlos. El capital: Crítica de la economía política. Trad. por Wenceslao

Roces. 3. vols. 3a. ed. México, Fondo de Cultura Económica, 1964. Vol. I. 769 págs. ${ }^{52}$

De tal manera, el modelo general de ficha o entrada bibliográfica común de estos tres textos es el siguiente: Apellido del autor, Nombre del autor. Título de la obra. Lugar de publicación, Editorial, fecha de publicación.

La Guía de López Valdés presenta una ficha más amplia, pero que también participa del mismo modelo, con la excepción de la coma entre el nombre del autor y el título, que en los otros manuales es un punto, y del uso de versales para el apellido del autor:

APELLIDOS DEL AutOR, Prenombre, Título. Subtítulo. 2a. ed., aum. Ed., introd y notas de Fulano. Ciudad, editorial, año. XXX + 000 pp. Ils. de Mengano. (Nombre de la colección, 00). ${ }^{53}$

Al final, el modelo que propone el manual de la UACJ tiene similitudes estilísticas con el manual anterior, aunque presenta algunas variantes menores: indica los datos de edición y reimpresión antes de referir la fecha, e invierte el orden de editorial y lugar de publicación:

${ }^{50}$ Bosch García, La técnica de investigación documental, 58.

${ }^{51}$ Torre Villar y Navarro de Anda, La investigación bibliográfica, 261.

${ }^{52}$ Galindo, Galindo y Torres-Michúa, Manual de redacción e investigación, 204.

${ }^{53}$ López Valdés, Guía de estilo editorial para obras académicas, 90. 
BACHELARD, Gastón, La poética del espacio (trad. Ernestina de Champourcin). FCE, Ciudad de México, 2a. ed., 8a. ri., 1975, 281 pp. [Breviarios, 183]. ${ }^{54}$

A partir de este modelo, ya sea implícito o explícito, estos cinco manuales construyen de manera muy similar los ejemplos y modelos para otras fuentes, como revistas o periódicos. Sin duda resultaría llamativa tanta coincidencia si no existiera una guía de este sistema, dada la complejidad del asunto. Podría pensarse, efectivamente, que se trata de un sistema marcado por el uso y la práctica, aunque el nivel de coincidencia entre los textos revisados confirma que es un sistema que está regulado con precisión y de manera explícita, como dijimos, por los lineamientos ISO 690.

Las variaciones encontradas, especialmente en la puntuación o en el uso de versales, responden a que el propio ISO se concibe como una guía flexible y no normativa. No deja de representar un problema que manuales dedicados a explicar y formar en los principios de investigación, ignoren u omitan que tras algunas de las prácticas editoriales y las convenciones más comunes no se haga una referencia clara y directa o la guía ISO como fuente de dichas prácticas y convenciones.

La mención explícita a las normas ISO, más allá de aprovechar su flexibilidad, permitiría resolver muchas de las dudas planteadas al revisar los manuales, y evitaría futuras confusiones. En primer lugar, se mostraría el carácter unificado de esta guía, con la tolerancia y flexibilidad que permite. Esto aportaría fiabilidad, ayudando a aclarar dudas al remitir a la fuente primaria, lo que mejoraría la accesibilidad, pues sin duda las normas ISO han mostrado su utilidad. Además, la posibilidad de dejar de recomendar el uso de los términos latinos, ayudaría a facilitar su uso. Así, en la medida en que se abandone el problema de comprensibilidad-derivado del uso de términos como sistema latino, hispánico o tradicional, que no remiten a ningún estilo o manual precisose ganará en claridad y en los factores señalados.

\footnotetext{
${ }^{54}$ Esquivel Carreón, Rodas Rivera y Salazar Quintana, Estilo Latino-humanístico, 80.
} 


\section{Conclusiones}

Tras lo expuesto, podemos aseverar que existe un grupo de pautas para referir fuentes, en documentos académicos, que utiliza de forma amplia los latinismos y la consignación de la información bibliográfica en notas a pie de página. Dichas pautas suelen considerarse de manera confusa un "sistema" que responde a los nombres de "latino", "hispánico" o "tradicional", al cual se le caracteriza por responder a una cuestión de usos y costumbres, pues carece de un manual o una institución que regule o actualice sus características y normas. Estos factores dificultan poderlo identificar como un sistema de referencia y manejo bibliográfico de manera plena, en particular si se compara con aquellos, como los mencionados APA, Chicago, Harvard, ISO o MLA, que sí cuentan con un manual, elaborado y revisado periódicamente por una institución identificada plenamente.

En otros casos, estas pautas no tienen un apelativo y se presentan falsamente como la única forma de citar y referir fuentes, producto de recoger la experiencia de investigadores versados en la materia. Esto hace creer que las normas son elaboradas por los autores de los manuales y que no las tomaron de ningún sistema previo, pues no citan la fuente de la cual se tomó la información. De esta forma, se crea la idea de que existe un sistema de referencias único o propio del mundo hispánico que, en ocasiones, se posiciona frente a los sistemas anglosajones, aunque comparta varios elementos estructurales con otros sistemas de referencia con notas a pie de página, como el caso de Chicago.

En realidad, las pautas presentadas en los manuales revisados siguen, en mayor o menor medida, la norma ISO 690, presentada en forma de directrices flexibles y variables (en términos estilísticos). Este factor explica las disimilitudes observadas entre los distintos manuales, pero también las grandes coincidencias en los lineamientos generales que la norma internacional dicta y estos documentos siguen. Más allá de pensar si los usos y costumbres fueron los que construyeron las normas ISO, tendrían que verse como la posibilidad de unificar y darle identidad a las prácticas que aún prevalecen para referir las fuentes con nota a pie, elemento central de los textos aquí revisados, sobre el cual Grafton señala que:

Citations in scientific works -as a number of studies have shown- do far more than identify the originators of ideas and the sources of data. They reflect the intellectual style of different national scientific communities, the pedago- 
gical methods of different graduate programs, and the literary preferences of different journal editors. They regularly refer not only to the precise sources of scientists' data, but also to larger theories and theoretical schools with which the authors wish or hope to be associated. ${ }^{55}$

El presente trabajo ha mostrado dichos fenómenos de carácter histórico, sobre la forma de citar, por medio de la revisión de las tendencias de algunos manuales que fueron importantes en la formación de los profesionales de las humanidades en México. Particularmente, al presentar algunos sesgos y carencias de esta tradición académica, se hace posible subsanar estas deficiencias y mejorar las prácticas existentes, partiendo de los aciertos de los textos analizados.

Ante la existencia de varios sistemas probados durante más de un siglo, consolidados y adaptados al quehacer actual de las humanidades, resulta ya insostenible seguir apelando a un supuesto sistema sin manual, sin un nombre claro ni una institución que lo regule. Continuar con la idea de un sistema "latino", "tradicional" o "hispánico" que no esté unificado ni sea fiable y accesible por medio de un manual de investigación académica elaborado a partir del consenso de los especialistas sólo contribuye a dificultar la formación de los investigadores y a crear confusiones bibliográficas.

\section{Referencias}

Asociación Española de Normalización y Certificación. Información y documentación. Directrices para la redacción de referencias bibliográficas y de citas de recursos de información. Madrid: AENOR, 2013.

Astey V., Luis. Procedimientos de edición para la Biblioteca Novohispana. México: El Colegio de México, 1985.

Besterman, Theodore. A World Bibliography of Bibliographies, and a Bibliographical Catalogue, Calendars, Abstracts, Digest, Indexes, and the Like. Ginebra: Societas Bibliographica, 1955.

Bosch García, Carlos. La técnica de investigación documental. 3a. ed. Caracas: Universidad Central de Venezuela, Instituto de Investigaciones Económicas y Sociales, 1972.

\footnotetext{
${ }_{55}$ Anthony Grafton, The Footnote. A Curious History (Cambridge, MA: Harvard University Press, 1999), 12-13.
} 
The Chicago Manual of Style. 17a. ed. Chicago: The University of Chicago Press, 2017.

El Colegio de México. "Lineamientos para publicar". Nueva Revista de Filología Hispánica. Acceso el 13 de enero de 2021. https://nrfh.colmex.mx/index. $\mathrm{php} / \mathrm{nrfh} /$ lineamientosparapublicar.

Esquivel Carreón, Guadalupe, Beatriz Rodas Rivera y Luis Carlos Salazar Quintana. Estilo Latino-humanístico. Redacción de textos académicos. Ciudad Juárez: Universidad Autónoma de Ciudad Juárez, 2017.

Galindo, Carmen, Magdalena Galindo y Armando Torres-Michúa. Manual de redacción e investigación. México: Grijalbo, 1997.

Gerould, Winfred Gregory, Donald B. Gilchrist, Wylis E. Wright, Daniel C. Haskell y Karl Brown. Bibliography Union Lists of Serials in Libraries of the United States and Canada. Nueva York, 1943.

Grafton, Anthony. The Footnote. A Curious History. Cambridge, MA: Harvard University Press, 1999.

Grenfell, David. Publicaciones periódicas y seriadas. Su tratamiento en las bibliotecas especializadas. Washington, DC: Unión Panamericana, 1962.

International Standard ISO 690: Information and Documentation - Guidelines for Bibliographic References and Citations to Information Resources. 3a. ed. Ginebra: International Organization for Standardization, 2010.

Lobato Domínguez, Javier. "El nuevo marco normativo en Gestión de documentos de archivo, las normas ISO / UNE: Guía para profesionales". arch-e. Revista Andaluza de Archivos 1 (mayo de 2009).

López Valdés, Mauricio. Guía básica de estilo editorial. México: Universidad Nacional Autónoma de México, Facultad de Filosofía y Letras, 2003.

López Valdés, Mauricio. Guía de estilo editorial para obras académicas. México: Ediciones del Ermitaño / Universidad Nacional Autónoma de México, Centro Regional de Investigaciones Multidisciplinarias, 2009.

Muñoz-Alonso López, Gemma. "Citación y referenciación en el ámbito de la filosofía: personalización de estilos internacionales mediante gestores bibliográficos". ÉNDOXA: Series Filosóficas 31 (2013): 211-252.

Muñoz-Alonso López, Gemma. "Tendencias actuales de citación en los trabajos de investigación filosófica". Investigación Bibliotecológica 41, núm. 20 (diciembre de 2006): 91-106.

Perelló, Mercedes, Margarita Pérez, Manuel Romero, Ella Suárez y Nicolás Vaughan. Manual de citas y referencias bibliográficas. Biblioteca del Editor. 
México: Universidad Nacional Autónoma de México / Universidad de Los Andes, 2018.

Real Academia Española. Libro de estilo de la lengua española según la norma panhispánica. Barcelona: Real Academia Española / Espasa, 2018.

Romero, Manuel. "Sistema latino". En Manual de citas y referencias bibliográficas: Latino, APA, Chicago, IEEE, MLA, Vancouver. 2a. ed. Edición de Manuel Romero, Ella Suárez, Nicolás Vaughan y Margarita Pérez, 89-102. Bogotá: Universidad de Los Andes, Vicerrectoría Académica, 2015.

Torre Villar, Ernesto de la, y Ramiro Navarro de Anda. La investigación bibliográfica, archivística y documental: su método. México: Universidad Nacional Autónoma de México, 2003.

Universidad Nacional Autónoma de México. "Colaborar en la revista". Revista de la Universidad de México. Acceso el 13 de enero de 2021. https://www. revistadelauniversidad. $\mathrm{mx} /$ publish.

Varossieau, W. W. A Survey of Scientific Abstracting and Indexing Services. La Haya: International Federation for Documentation (FID), 1949.

Winchell, Constance M. Guide to Reference Books. 7a. ed. Chicago: Chicago American Library Association, 1951. $\mathrm{bg}$ 